\title{
Ultrasound For Critical Care Physicians: Neutropenic Patient With Fever and Shortness of Breath
}

\author{
Erik Kraai MD \\ Michel Boivin MD \\ Division of Pulmonary / Critical Care and Sleep \\ University of New Mexico \\ Albuquerque, NM
}

A 63 year old female with a history of acute myelogenous leukemia presents with shortness of breath, fever and hypotension to the ICU. She is in septic shock on norepinephrine, and has been treated on the oncology unit with vancomycin, cefepime, acyclovir and voriconazole. She has been neutropenic for 1 month. The patient develops a progressive right lower chest opacity. This opacity has progressed in spite of antibiotics and antifungals. The portable AP chest radiograph is presented below (Figure 1).

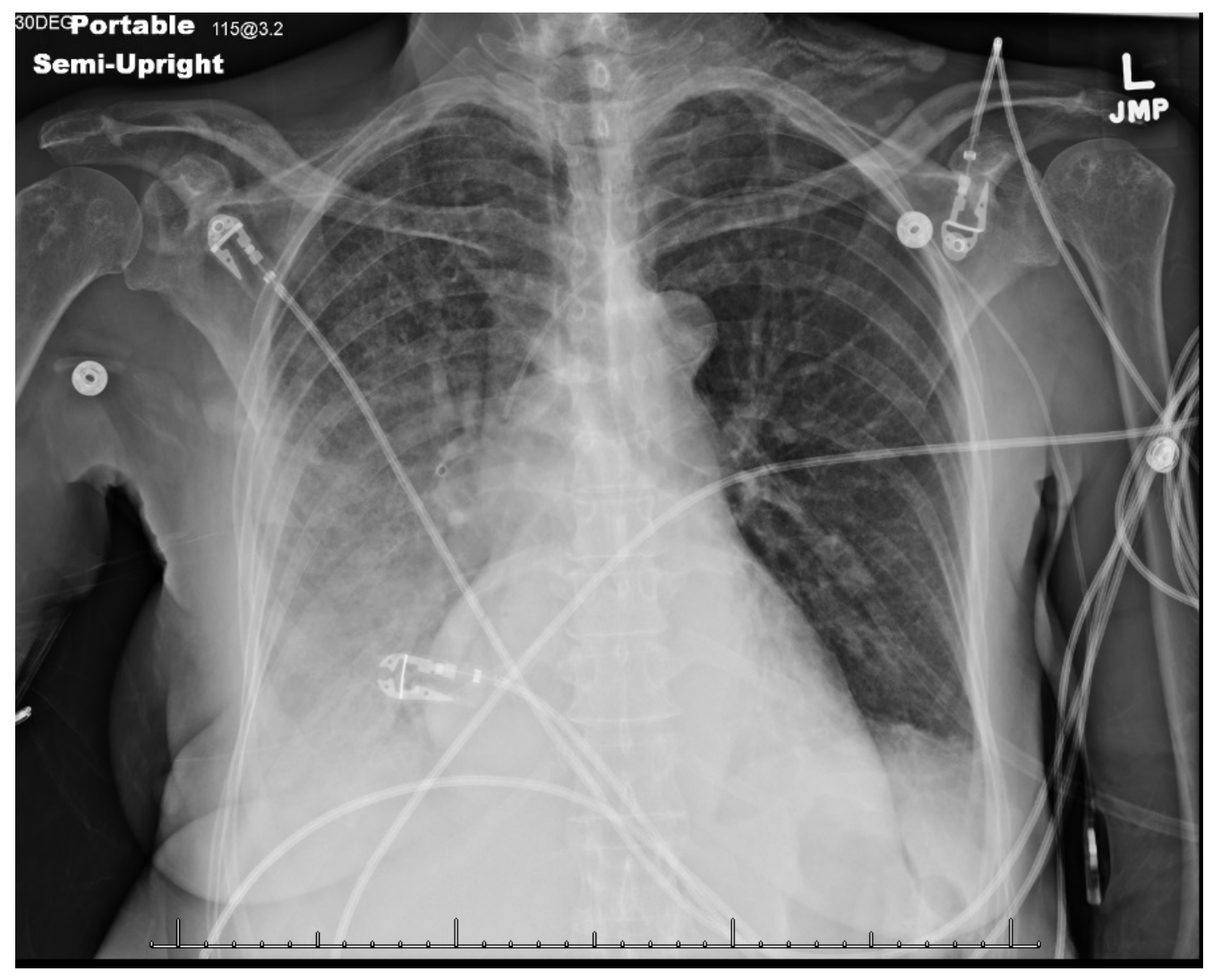

Figure 1. Portable AP of chest.

An ultrasound of the right chest was performed for further evaluation of the opacity (figure 2). 


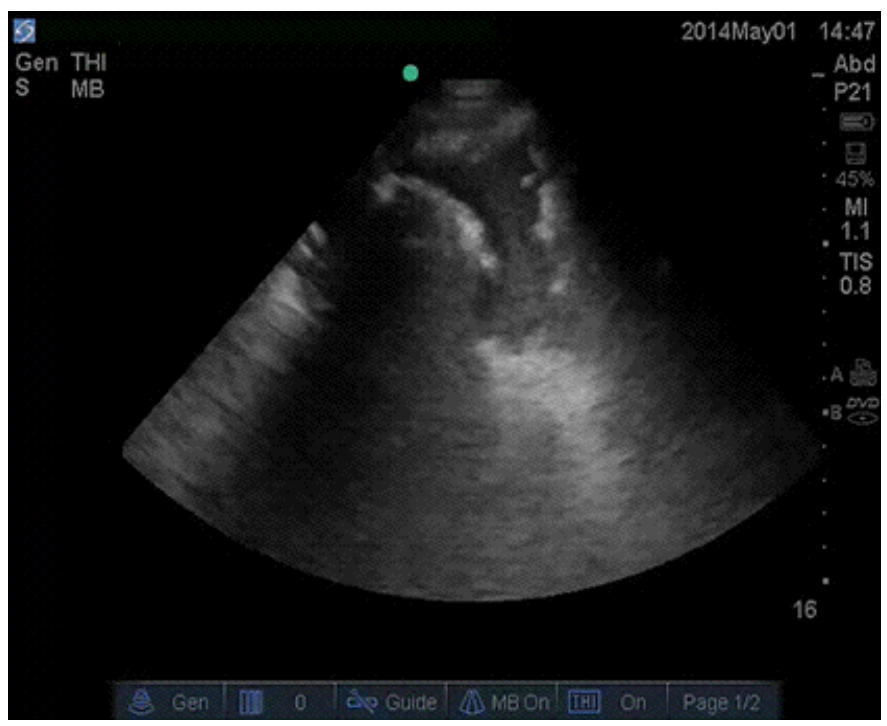

Figure 2. Static ultrasound of right hemithorax.

Question: What pathology does the ultrasound reveal in the right hemithorax?

1. Air filled cavity

2. Chest wall abscess

3. Fractured ribs

4. Pleural effusion and suspected empyema

5. Simple consolidation 


\section{Correct! \\ 1. Air filled cavity.}

The ultrasound reveals the presence of an air filled cavity surrounded by consolidation (figure 3).

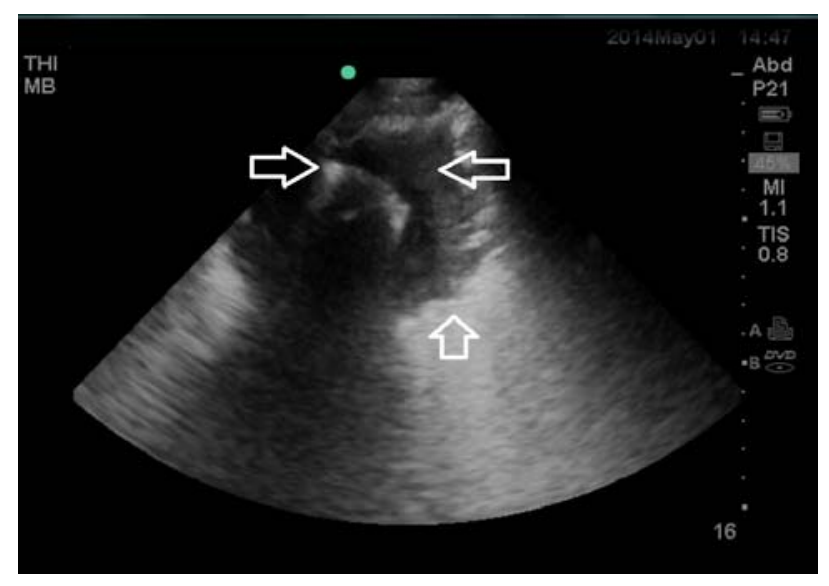

Figure 3. Static image of the ultrasound of the right hemithorax.

The areas of consolidated lung are marked with a left pointing arrow, demonstrating isoechoic and hypoechoic areas. The hyperechoic air filled center of the cavity (right pointing arrow) demonstrates posterior shadowing due to the impenetrability of ultrasound waves to air. Distal to the cavity, hyperechoic, air-filled lung (up-pointing arrow) is demonstrated at the edges of the consolidation / cavity. In this case ultrasound demonstrated the presence of an evolving cavity before it was appreciated on plain radiography. The patient underwent a CT scan of the chest (figure 4) the following day which confirmed the presence of the consolidation and cavity.

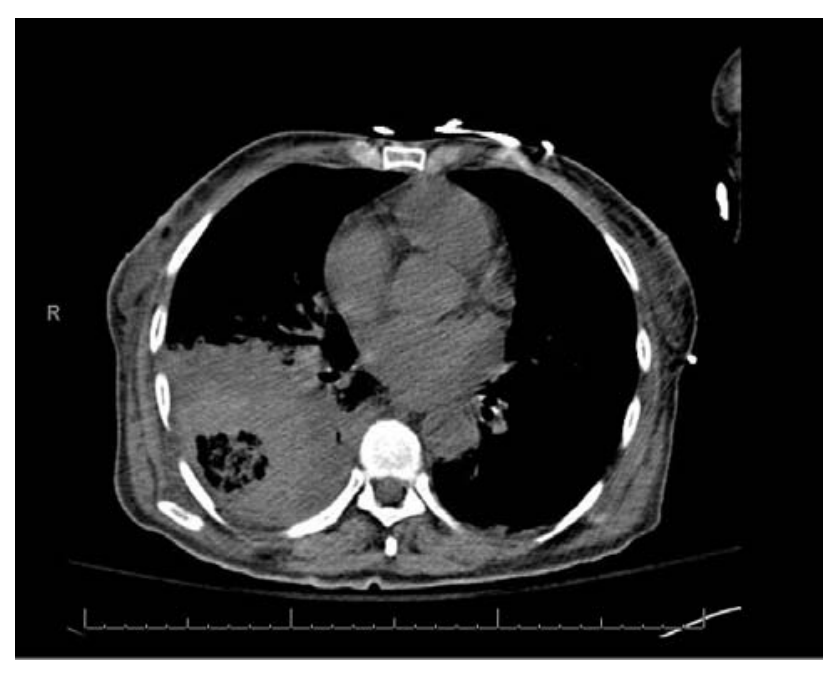

Figure 4. Representative image from the thoracic CT scan showing consolidation and air-filled cavity. 
The CT was read as most compatible with angioinvasive fungal infection. The patient was switched to amphotericin B. Unfortunately, the patient continued to deteriorate. She opted for comfort care before definitive diagnosis could be made.

There are few descriptions of cavities diagnosed by ultrasound in the medical literature. But, in this case, the constellation of ultrasound findings clearly demonstrated this to be the nature of the lesion early in the course. The case demonstrates the utility of ultrasound in the evaluation of lung and pleural disease in the intensive care unit. 